\title{
The application of multiobjective programming with regard to the problem of bandwidth distribution
}

\author{
Xu Baoji ${ }^{1 \text {, a }}$, Sun Zhihong ${ }^{1}$, Liu Gang ${ }^{1}$ and Yang Guikao ${ }^{1}$ \\ ${ }^{1}$ Basic Department of Xu zhou Air Force College Xuzhou, China \\ axbj_1008@163.com
}

\begin{abstract}
Keywords: The multiple objectives planning model; Bandwidth distribution; Objective functions;
\end{abstract} graphical method.

\begin{abstract}
The application of multiobjective programming with regard to the problem of bandwidth distribution is very important. For the remote places, the uneven-distribution and the fluidity of users will not lead to regional dense population. Therefore, the large-scale repeaters with wide radiation range will serve the areas well. the objective of the model is to find out the maximum of the communication definition and accessibility. The constraint conditions are determined by the relationship of definition, accessibility and the repeaters' number. the model is solved through graphical method. Seven reasonable installing plans of repeaters are obtained. Different areas should choose the right plan in the light of local conditions. The rationality is testified through simulation.
\end{abstract}

\section{Introduction}

The wireless communication has played a significant and indispensable role in daily lives. Countless repeaters and users have formed an enormous network which makes the transfer of data and the exchange of information come true. However, the limited frequency spectrum can not fulfill the daily-increasing demand for information exchange any more. To relieve this problem, the only way is to build a rational information exchange network. Different areas have distinct demands for the information exchange. According to the user population in a unit area, the discussion should be about the densely populated and sparsely populated areas respectively.

For the remote places, there exists no dense population, and the requirement for bandwidth is not strict. Therefore, the large-scale repeaters with wide radiation range will serve the areas well. The only factor influencing the regional communication is the number of repeaters. If there are too few repeaters, each repeater has to undertake too many users which will lead to communication delay; on the other side, due to the upper limitation of bandwidth, if the number of repeaters is too much, the spectrum bandwidth shared by each repeater would be relatively narrow which is the reason of mutual interference during communication. The key point is to find out the balance between the definition and accessibility of communication.

\section{Multiple Objectives Planning Model}

In a remote or sparsely populated area, the large-scale repeaters will be utilized to fulfill the objective of long distance information transmission. The quality of communication service is determined by two factors: communication definition and accessibility. If there are too few repeaters, each repeater has to undertake too many users which will lead to communication delay; on the other side, due to the upper limitation of bandwidth, if the number of repeaters is too much, the spectrum bandwidth shared by each repeater would be relatively narrow which is the reason of mutual interference during communication.

Based on the discussion above, supposing the number of repeaters installed in this area is $x$, using $\eta$ to denote the communication definition and $\delta$ to denote communication accessibility, then the objective function of the multiple objectives planning can be obtained:

$$
\max (\eta, \delta)^{T}
$$


The constraint conditions of the function can be determined through the analysis of communication definition and accessibility.

The PL (private line) and the electromagnetic signal from transmitting end are combined by the "Continuous Tone-Coded Squelch System" (CTCSS) ${ }^{[1]}$ in the repeater. The receiving end only receives signals with specific PL, as a result, users shared the same repeater will not interfere each other. The only interference is from a neighbor repeater whose signals are transmitted through the same PL. In other words, the user-to-user interference is simplified as the interference among repeaters thanks to the CTCSS. Whether the receiving end will pick up signals form other transmitting ends depends on the given PL of the receiving end. The interference intensity is up to the similarity of signal frequency of receiving and transmitting ends, i.e., the adjacent-channel interference ${ }^{[2]}$.

The frequency difference between the receiving end and the transmitting end is $\Delta f$, in the adjacent-channel interference. According to the simulation results of [2], the interference degree caused by $\Delta f$ approximately representing the normal distribution ${ }^{[3]}$ whose expectation is 0 and variance is $\mathrm{b}$. Here $\mathrm{b}$ is a parameter which is determined by the properties of repeater itself.

Supposing $\Delta f \sim N(0, b)$, defining the definition of the receiving end $i$ as

$\eta_{i}=1-F(\Delta f)$.

The central frequency of repeater $i$ is $f_{i}, i=1,2,3 \cdots x$ the central frequency difference between the repeater $i$ and its neighbor repeater is $\Delta f_{i}, \quad i=1,2,3 \cdots x-1$.

The communication definition of the given area:

$\eta=\min \left(\eta_{i}\right)$,

When $\Delta f=\min \left(\Delta f_{i}\right)$, obtaining the maximum. When $x$ is invariable, if $\Delta f_{i}$ are equal, $\eta$ gets its maximum. The frequency difference of frequency-adjacent repeaters is:

$\Delta f=\frac{W}{x} M H z$.

According to the statistics ${ }^{[4]}$, the probability density function of normal distribution $N(0, b)$ is

$$
F(\Delta f)=\frac{1}{\sqrt{2 \pi} b} e^{\frac{\Delta f^{2}}{2 b^{2}}} \text {. }
$$

Apart from the signal interferences of the repeater nearby the one connecting to the receiving end, there are also other repeaters can lead to interferences. Based on the given question, the research significance is about the relationship between the frequency difference and the interference. All interferences can be replaced by the interferences of another repeater nearby the one connecting to the receiving end. I.e., other interferences can not change the result. Therefore, according to the expressions (2) (3) (4) (5), the relationship between the definition $\eta$ and the number of repeaters $x$ can be expressed as:

$$
\eta=1-\frac{1}{\sqrt{2 \pi} b} e^{\frac{x^{2}}{2 b^{2} w^{2}}}
$$

There are two unknown parameters $W$ and $b$ in the expressions. The value of $W$ can usually be obtained on the basis of the given area conditions, while $b$ is up to the receiving end, that is the receiving range $K$ of user. The value of $b$ can be acquired as follows:

When the difference between noise frequency and the given frequency of the receiving end is out of the receiving range, the interference degree will be too little to be considered, though theoretically the interference exists. I.e., when $\Delta f=K, F(\Delta f)<\varepsilon, \varepsilon$ is a rather small number who can reflect the precision of the model.

$$
\frac{1}{\sqrt{2 \pi} b} e^{\frac{K^{2}}{2 b^{2}}}<\varepsilon .
$$

The expressions (6) (7) could be the constraint conditions of definition degree, in the multiple 
objectives planning model.

Whether the users sharing the same repeater need to use the PL, this probability represents the Poisson distribution ${ }^{[5]}$ whose parameter is $\lambda$. Thus the probability for every repeater can work normally is

$$
P_{i}=\sum_{k=0}^{M} \frac{\lambda_{i}^{k} e^{-\lambda_{i}}}{k !},
$$

The $M$ means the number upper limitation of PL. There are two parameters in the expression. $M$ is a property of the repeater which is determined by the repeater itself. $\lambda$ is the expectation value of the Poisson distribution Based on the law of large numbers, its value could approximately be:

$\lambda \approx n p$,

$p$ is the probability for a user occupying the PL at some time. $n$ is the number of users sharing the same repeater.

The communication accessibility of the given area is:

$\delta=\min \left\{P_{i}\right\}$,

If the numbers of people connecting each repeater are equal, $\delta$ gets its maximum. And

$n=\frac{R}{x}$.

R stands for the total number of users. According to the expressions (8) (9) (10) (11), there obtained the relationship between the communication accessibility $\eta_{2}$ and the number of the repeaters $x$ in the given area:

$$
\delta=e^{-\frac{R p}{x}} \sum_{k=0}^{M} \frac{(R p)^{k}}{x^{k} k !} .
$$

The expression (12) could be the constraint condition of communication accessibility, in the multiple objectives planning model.

The Determination of Model:

$$
\begin{aligned}
& \max (\eta, \delta)^{T} \\
& \text { s.t. }\left\{\begin{array}{l}
\eta=1-\frac{1}{\sqrt{2 \pi} b} e^{\frac{x^{2}}{2 b^{2} W^{2}}}, \\
\frac{1}{\sqrt{2 \pi} b} e^{\frac{K^{2}}{2 b^{2}}}<\varepsilon, \\
\delta=e^{-\frac{R p}{x}} \sum_{k=0}^{M} \frac{(R p)^{k}}{x^{k} k !}, \\
0 \leq \eta \leq 1, \\
0 \leq \delta \leq 1 .
\end{array}\right.
\end{aligned}
$$

\section{The Simulation of Model}

The designed multiple objectives planning model has one variable which can be solved through graphical method. Setting the precision degree $\varepsilon=0.001$, the probability for single user asking for communication $p=0.5$, the picture can be obtained. 


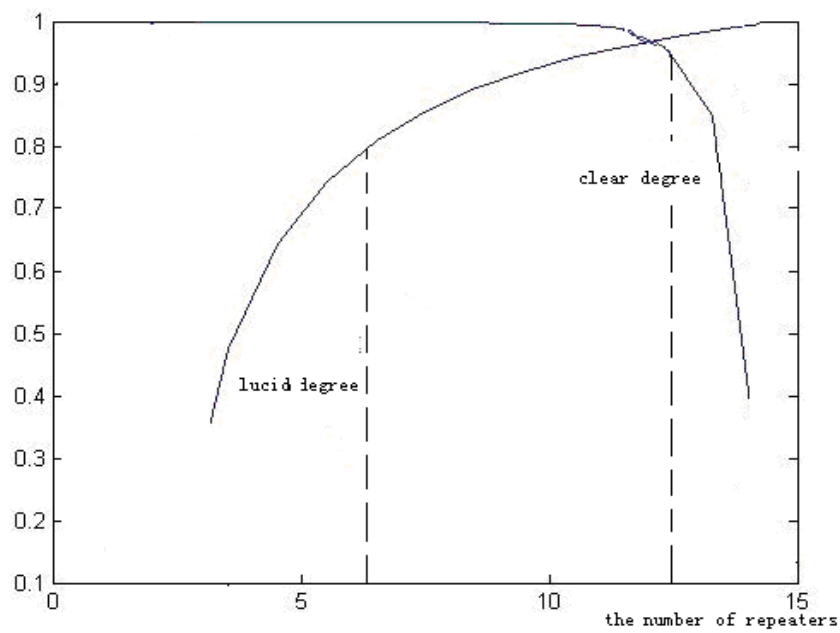

Figure $1(\xi=0.001, p=0.5)$

In the picture, it can be seen that, the communication definition and accessibility is of high quality when the number of repeaters is from 6 to 12. If a high definition wanted to be guaranteed, the number of repeaters should be reduced; while the increase of repeaters will lead to a higher accessibility. The decision can be made in the light of the real demands of different areas, as well as the factor of price, and so on.

\section{Conclusion}

On the basis of real situations in real life, the research resolves the problems on the bandwidth distribbtion of wireless communication. On the premise of a overall consideration of several factors which can influence the communication quality, the paper has built a comprehensive but simple model. Then the reasonable distribution plan is obtained. A great number of detailed and reliable materials acquired from internet and library play an important part in the tests of the model.

\section{References}

[1] Dorota Rabczuk. The Micro-Controller System for Remote Control of the Functions of RadioCommunication system with CTCSS and Sell-Call Signaling[C]. Lviv: Proe od IEEE TCSET, 2004:505-506.

[2] HAAS H,MCLAUGHLIN S.A Derivation of the PDF of Adjacent Channel interference in a Cellular System[J]. IEEE Communications Letters,2004,18(2):102-104.

[3] From Wikipedia, the free encyclopedia. Normal distribution . http://en.wikipedia.org/wiki/Normal_distribution .2011-02-13.

[4] A.C.Cohen. Estimating the parameter in a conditional Poisson distributions. Biometries 16:203-211(1960) 